\title{
A note on non-uniform points for projections of hypersurfaces
}

\author{
Maria Gioia Cifani ${ }^{1}\left[\right.$ Diccardo Moschetti ${ }^{2}$
}

Received: 13 December 2021 / Accepted: 2 February 2022 / Published online: 27 February 2022

(c) The Author(s) 2022

\begin{abstract}
Let $X$ be an irreducible, reduced complex projective hypersurface of degree $d$. A uniform point for $X$ is a point $P$ such that the projection of $X$ from $P$ has maximal monodromy. We extend and improve some results concerning the finiteness of the locus of non-uniform points for projections of hypersurfaces obtained by the authors and Cuzzucoli (Ann. Mat. Pura ed Appl. 1923, 1-18 (2021)) only for $P$ not contained in $X$.
\end{abstract}

\section{Introduction}

Let $X$ be an irreducible, reduced complex projective hypersurface of dimension $n \geq 1$ and degree $d$. Fixing a point $P \in \mathbb{P}^{n+1}$, we can study the linear projection map $\pi_{P}: \mathbb{P}^{n+1} \backslash\{P\} \rightarrow \mathbb{P}^{n}$. If we restrict $\pi_{P}$ to $X$, we get a finite map of degree $d-1$ or $d$, depending on $P$ being a smooth point of $X$ or being outside $X$. It is well known that the monodromy group $M\left(\left.\pi_{P}\right|_{X}\right)$ of the projection (cf. Definition 2.10 below) is a subgroup of the symmetric group $S_{d}$ if $P \notin X$ or $S_{d-1}$ if $P \in X^{\mathrm{sm}}$. A consequence of the classical uniform position principle is that, when $P$ is general in $\mathbb{P}^{n+1}$, then $M\left(\left.\pi_{P}\right|_{X}\right)$ coincides with $S_{d}$. This motivates the following definition.

Definition 1.1 A point $Q \in \mathbb{P}^{n+1} \backslash X$ is called outer uniform point if $M\left(\left.\pi_{Q}\right|_{X}\right)$ is isomorphic to $S_{d}$. A point $Q \in X^{\mathrm{sm}}$ is called inner uniform point if $M\left(\left.\pi_{Q}\right|_{X}\right)$ is isomorphic to $S_{d-1}$. We will denote by $\mathcal{W}(X)^{\text {in }}$ (resp. $\mathcal{W}(X)^{\text {out }}$ ) the locus of points $Q \in X^{\mathrm{sm}}$ (resp. $Q \in \mathbb{P}^{n+1} \backslash X$ ) which are not-uniform. Furthermore, $\mathcal{W}(X)$, also called the locus of non-uniform points will be the union of $\mathcal{W}(X)^{\text {in }}$ and $\mathcal{W}(X)^{\text {out }}$.

$凶 \quad$ Maria Gioia Cifani

mariagioia.cifani@uniroma3.it

Riccardo Moschetti

riccardo.moschetti@unito.it

1 Department of Mathematics and Physics, Roma 3 University, Largo San Murialdo 1, 00146 Rome, Italy

2 Department of Mathematics 'G. Peano', University of Turin, Via Carlo Alberto 10, 10123 Turin, Italy 
The following conjecture extends [4,Conjecture 1.7] to inner non-uniform points:

Conjecture 1.2 Let $X$ be a complex irreducible, reduced projective hypersurface of dimension $n \geq 1$. The locus $\mathcal{W}(X)$ is finite unless $X$ is a cone.

The case of $n=1$ has been addressed in Proposition 3.4 and Theorem 3.5 of [18]. Notice that if $X$ is an irreducible curve, $X$ is not a cone and so the conjecture holds. Other evidence for the conjecture can be found in the context of Galois points. We say $Q \in \mathbb{P}^{n+1}$ is a Galois point if the field extension associated with the projection $\left.\pi_{Q}\right|_{X}$ is a Galois extension. Galois points are a particular case of non-uniform points, and have been extensively studied in $[8,9,21]$. In particular, Theorem 1.1 and Theorem 1.2 of [10] show that Conjecture 1.2 holds for Galois points when $X$ is a normal hypersurface. In a slightly different direction, it has been proven in [6] and [3] that the conjecture holds true for $X$ general, with $\mathcal{W}(X)$ empty. The case of $X$ being a smooth hypersurfaces has been proved in [7, Theorem 1.1] for $n=2$ and in [4, Theorem 1.3] in higher dimension, both for outer non-uniform points.

The aim of this paper is twofold: we first extend the results of [4] to inner nonuniform points, and then we study the possible counterexamples to the conjecture, showing that the only possible class of hypersurface which could provide a counterexample to the conjecture is given by hypersurfaces $X$ in $\mathbb{P}^{n+1}$, which are not cones, for which there is a $\mathbb{P}^{k}(0<k<n)$, such that $X \cap \mathbb{P}^{k+1}$ is reducible for every $\mathbb{P}^{k+1}$ which contains $\mathbb{P}^{k}$. This is done in Corollary 3.10 and improves [4,Remark 4.11].

The key result is a generalisation of [4, Theorem 1.2]:

Theorem 1.3 Let $X$ be a complex irreducible, reduced hypersurface of $\mathbb{P}^{n+1}, n \geq 2$. Then $\mathcal{W}(X)$ is contained in a finite union of linear spaces of codimension 2 in $\mathbb{P}^{n+1}$.

The proof mainly relies on the study of the focal locus of certain families of lines. While the core results were already developed in [5] and [4], the difficulty in generalizing the setting to inner non-uniform point relies upon carefully choosing the family. Moreover, we were able to improve [4, Theorem 1.2] thanks to a local study of the tangent cone to singular points of the hypersurface (Lemma 2.9).

As a consequence of the main theorem, we can show the finiteness of the locus of non-uniform points for smooth hypersurfaces:

Theorem 1.4 Assume $X$ is a complex smooth projective hypersurface or a general projection of a smooth variety to a hypersurface. Then, the locus $\mathcal{W}(X)$ is finite.

We also get a generalisation of the result in [10], which also proves the conjecture when $X$ has prime degree.

Theorem 1.5 Assume $X$ is an irreducible and reduced hypersurface in $\mathbb{P}^{n+1}$ which is not a cone. Then, the number of Galois points is finite. If $X$ has also prime degree (resp. $\operatorname{deg}(X)-1$ is prime), then $\mathcal{W}(X)^{\text {out }}$ (resp. $\left.\mathcal{W}(X)^{\text {in }}\right)$ is finite.

In the following, we will always work on the field $\mathbb{C}$ of complex numbers. For a variety $X$ we will denote by $X^{\text {sing }}$ its singular locus, and by $X^{\text {sm }}$ the locus $X \backslash X^{\text {sing }}$. 


\section{Preliminaries}

\subsection{Families of lines and focal loci}

A family $\mathcal{X}$ of lines in $\mathbb{P}^{n+1}$ can be parametrized by an integral subscheme $S$ of the Grassmannian $\mathbb{G}\left(1, \mathbb{P}^{n+1}\right)$. We can describe it by the following diagram

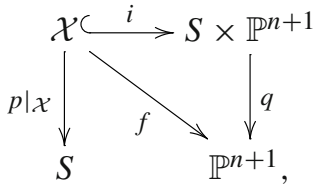

where the map $i$ is the inclusion and $p, q$ are the projections on the first and second factors, respectively.

Definition 2.1 The focal sheaf is the sheaf over $\mathcal{X}$ associated with the kernel of the map differential $\mathrm{d} f$. The support of this sheaf is called the focal locus of the family $\mathcal{X}$.

Definition 2.2 A family $\mathcal{X}$ of lines in $\mathbb{P}^{n+1}$ is called filling family if the dimension of the base $S$ is $n$ and the map $f=i \circ q$ is dominant.

We need some results concerning relationships between the focal locus and the property of being a filling family.

Lemma 2.3 ( [5], Proposition 4.1) Let $\mathcal{X}$ be a filling family of lines in $\mathbb{P}^{n+1}$ and let $s$ be a general point of $S$. Then the focal locus in the fibre $\ell_{s}$ consists of $n$ points counted with the right multiplicity.

The following result dates back to Segre, in [19]:

Lemma 2.4 Let $\mathscr{X}$ be a filling family of lines in $\mathbb{P}^{n+1}$ and assume that $\mathscr{X}^{\prime}$ is a subfamily consisting of lines all passing through a point $P$. If the dimension of the base of $\mathscr{X}^{\prime}$ is $k$, then $P$ has multiplicity $k$ as a focal point of $\mathscr{X}$.

When the integer $k$ used in the previous lemma is greater or equal than $1, P$ is also called a fundamental point. We recall the following result on filling families of lines given by the join of two subvarieties.

Lemma 2.5 [4,Lemma 3.8] Let $F$ be a subvariety of $\mathbb{P}^{n+1}$ of codimension 2, and $\mathscr{C} \nsubseteq F$ be a curve not contained in a $\mathbb{P}^{n-1}$. Assume that the family $\mathcal{X}$ of lines joining $\mathscr{C}$ and $F$ is filling. Then $F$ is linear.

\subsection{Intersection of lines with a hypersurface}

Consider a degree $d$ integral hypersurface $X$ contained in $\mathbb{P}^{n+1}$, and a line $\ell \nsubseteq X$. Denote by $P_{1}, \ldots, P_{k}$ the points in $X \cap \ell$, and by $m_{i}$ the multiplicity of $P_{i}$ in $X \cap \ell$. We have that that $\sum m_{i}=d$. 
Definition 2.6 We call the contact order of $\ell$ with $X$ at $P_{i}$ the number $m_{i}-1$, and we denote it by $\operatorname{ord}_{P_{i}}(\ell \cap X)$.

The line $\ell$ is transverse to $X$ at $P_{i}$ if ord $_{P_{i}}(\ell \cap X)=0$, and tangent to $X$ at $P_{i}$ if $\operatorname{ord}_{P_{i}}(\ell \cap X) \geq 1$. In the case of higher contact order, i.e. $\operatorname{ord}_{P_{i}}(\ell \cap X) \geq 2$, we say that the line $\ell$ is an asymptotic tangent to $X$ at $P_{i}$. The line $\ell$ is called bitangent to $X$ at two points $P_{i} \neq P_{j}$, if $\ell$ is tangent to $X$ at both points $P_{i}, P_{j}$. We say that $\ell$ is a simply tangent if there is a unique tangent point $P_{i} \in \ell \cap X$ with $\operatorname{ord}_{P_{i}}(\ell \cap X)=1$ and $\ell$ is transverse to $X$ for all the other $P_{j} \neq P_{i}$ in $\ell \cap X$. We can classify such behaviours by means of the following datum:

$$
\beta(\ell):=\sum_{P_{i} \in X \cap \ell} \operatorname{ord}_{P_{i}}(\ell \cap X) .
$$

We know that $\beta(\ell)=0$ if and only if $\ell$ is transverse to $X$ at all the $P_{i}, \beta(\ell)=1$ if and only if $\ell$ is simply tangent to $X$, and $\beta(\ell)>1$ if and only if $\ell$ is multi-tangent to $X$, namely is at least bitangent or asymptotic tangent to $X$ at some points.

Lemma 2.7 ([4], Proposition 4.1) Let $X \subset \mathbb{P}^{n+1}$ be an integral hypersurface, and $\mathcal{X}$ be a filling family of lines in $\mathbb{P}^{n+1}$. Assume the general line $\ell \in \mathcal{X}$ is tangent to $X$ at a general point $P$. Then, the point $P$ is a focus on $\ell$. If the contact order of $\ell$ with $X$ at $P$ is at least 2, then the multiplicity of $P$ as a focus on $\ell$ is at least 2.

We now prove a result using a local computation on the tangent cone that we will use in the proof of the main Theorem. First, we recall the definition of the tangent cone.

Definition 2.8 [14, Lecture 20] Consider a hypersurface $X \subset \mathbb{P}^{n+1}$ and a point $P \in X$. Choose an affine neighborhood of $P$, where $P$ is the origin. Here $X$ is described by the vanishing of a certain polynomial $f:=f_{m}+f_{m+1}+\cdots$, where $f_{k}$ is homogeneous of degree $k$, and $m$ is the smallest integer such that $f_{m}$ is not identically zero. The tangent cone to $X$ at the point $P$, denoted by $C_{P}(X)$, is the hypersurface of degree $m$ in $\mathbb{P}^{n+1}$ described by $\left\{f_{m}=0\right\}$.

Lemma 2.9 Let $P$ be a singular point of a reduced and irreducible hypersurface $X \subset \mathbb{P}^{n+1}$. Consider a hyperplane $H$ containing $P$ and a line $\ell \subset H$. If $\ell \in C_{P}(X)$, then there is a component $Y$ of $X \cap H$ such that $Y^{\mathrm{red}}$ is tangent to $\ell$ at $P$.

Proof We restrict ourselves to a general $K \cong \mathbb{P}^{2}, \ell \subset K \subset H$. We know that $\ell$ is one of the lines of $C_{P}(X) \cap K$. Since the tangent cone $C_{P}(X)$ describes $X$ locally around $P, C_{P}(X) \cap K$ describes $X \cap K$ locally around $P$, so there exist a component $Y_{K}$ of $X \cap K$ such that $Y_{K}^{\text {red }}$ is tangent to $\ell$ at $P$ in $K$. As a consequence, we can choose as $Y$ any component of $X \cap H$ containing $Y_{K}$.

\subsection{Monodromy group of projections}

Let $\pi: X \rightarrow Y$ be a generically finite, dominant morphism of degree $d$, with $X$ and $Y$ irreducible projective varieties. The morphism $\pi$ induces a map

$$
\mu: \pi_{1}(U, y) \rightarrow \operatorname{Aut}\left(\pi^{-1}(y)\right) \simeq S_{d}
$$


where $U$ is a Zariski open subset of $Y$ over which $\pi$ is étale and $y \in U$ is a point.

Definition 2.10 The image $M(\pi)=\mu\left(\pi_{1}(U)\right)$ is called monodromy group of $\pi$.

The monodromy group is isomorphic to the Galois group ( $[13, \mathrm{Sec} I])$, i.e. the Galois group of the field extension $K / k(Y)$, where $K$ is the Galois closure of the field extension $k(X) / k(Y)$. In particular, the monodromy group does not depend on the choice of the open set $U$. The group $M\left(\pi_{P}\right)$ is a transitive subgroup of the symmetric group since $X$ is irreducible. We recall some standard facts on transitive permutation groups (see for instance [15, Ch.8]).

Proposition 2.11 Let $G$ be a transitive subgroup of $S_{d}$. Then the following hold

- if $G$ is finitely generated and its generators are all transpositions but one, then $G=S_{d}$

- if $G$ is primitive and contains a transposition then $G=S_{d}$.

Remark 2.12 A map $\pi_{P}$ is decomposable if it factors non birationally over a open subset, see [18, Definition 2.1]. If $\pi_{P}$ is indecomposable, then the monodromy group $M\left(\pi_{P}\right)$ is primitive, see [18, Remark 2.2].

The following Bertini-type result will be used various times in the following. It can be useful for instance to find a set of generators for the monodromy group.

Proposition 2.13 Let $X$ be an irreducible variety in $\mathbb{P}^{n+1}$ of dimension $n \geq 2$. Let $H \simeq \mathbb{P}^{2}$ be a general plane and $X_{H}:=X \cap H$. Then, for a point $P \in H$ we have

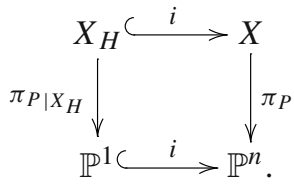

As a consequence we have $M\left(\pi_{P \mid X_{H}}\right) \leq M\left(\pi_{P}\right)$, which in term of non-uniform points gives that

$$
\mathcal{W}(X) \cap H \subseteq \mathcal{W}\left(X_{H}\right)
$$

The construction of the monodromy group associates a permutation to a generator of the fundamental group, which, thanks to the previous proposition can be associated with a branch point. The following proposition describes the cycle structure of the permutation depending on the structure of the fibre of the projection.

Proposition 2.14 [18, Prop. 2.5] If $\sum_{j=1}^{k} m_{j} P_{j}$ denotes the scheme theoretic fibre of $\pi$ over a branch point, then the associated permutation have a cycle structure $\left(m_{1}, \ldots, m_{k}\right)$.

In particular, simple branch points correspond to transpositions in the monodromy group [13, Lemma, pag. 698]. 
Remark 2.15 When $P \in X^{\mathrm{sm}}$ the map $\left.\pi_{P}\right|_{X}$ is rational and not defined on $P$. We compose it with the blow up $v: B l_{P} X \rightarrow X$ to get a morphism $B l_{P} X \stackrel{\pi}{\rightarrow} \mathbb{P}^{n}$ and the monodromy of $\left.\pi_{P}\right|_{X}$ coincides with the monodromy of $\pi$. In particular, a line bitangent to $X$ being simply tangent at $P$ and in another point is associated to a transposition in the monodromy group of the projection $\pi_{P}$.

By combining Propositions 2.11 and 2.14 we get the following property of nonuniform points in the case of planar curves, that we will use in the proof of the main results.

Lemma 2.16 Let $C \subset \mathbb{P}^{2}$ be an irreducible and reduced curve and. If $P \in \mathcal{W}(C)$ is a non-uniform point, then there are at least two lines multi-tangent to $C$ passing through $P$.

\section{The locus of non-uniform points}

This section is devoted to the proof of the main results of this paper. As a quick preamble, we recall that there are two differences with respect to the arguments of [4]: first, [4, Theorem 1.2] is improved, removing a rather technical case. This is a step forward in the direction of proving Conjecture 1.2, and also simplify the proofs of Theorems 1.4 and 1.5. Second, all the results are generalized also to $\mathcal{W}(X)^{\text {in }}$ : we now state them with $\mathcal{W}(X)$, which is the union of $\mathcal{W}(X)^{\text {out }}$ and $\mathcal{W}(X)^{\text {in }}$, and when it is required, we handle the two cases separately in the proofs.

We will need to work with a family of lines that are multi-tangent to $X$. For the technical part of the proof, we will need to select an appropriate subfamily, which we will call $\mathcal{V}(X)$. It will be related to the lines corresponding to generators of the non-uniform monodromy groups as in Proposition 2.14. We will denote by $\mathcal{V}(X)_{P}$ the family of multi-tangent lines to $X$ passing through $P$ which are still multi tangent at $X$ after removing the potential contribution from the point $P$. In the notation of Sect. 2.2, consider a line $\ell$ multi-tangent to $X$, so $\beta(\ell)>1$. If $P \notin X \cap \ell$, then trivially $P$ has no contribution in $\beta(\ell)$, so $\ell \in \mathcal{V}(X)_{P}$. If $P \in X \cap \ell$, then we have $\ell \in \mathcal{V}(X)_{P}$ if

$$
\sum_{P_{i} \in X \cap \ell, P_{i} \neq P} \operatorname{ord}_{P_{i}}(\ell \cap X)>1 .
$$

Definition 3.1 Let $\mathcal{V}(X) \subset \mathbb{G}\left(1, \mathbb{P}^{n+1}\right)$ be the family of lines in $\mathbb{P}^{n+1}$ obtained by the union of $\mathcal{V}(X)_{P}$ for $P \in \mathcal{W}(X)$. If $\mathscr{C} \subset \mathbb{P}^{n+1}$ is an algebraic set, we will denote by $\mathcal{V}_{\mathscr{C}}$ the union of $\mathcal{V}_{P}(X)$ for $P \in \mathscr{C}$.

We want to apply the results about the focal loci of the family $\mathcal{V}(X)_{\mathscr{C}}$, when $\mathscr{C}$ is a curve contained in $\mathcal{W}(X)$. We remark that by our definition of tangency, the lines of $\mathcal{V}(X)_{\mathscr{C}}$ are not contained in $X$. The fact that the family is non-empty comes from the next two lemmas.

Lemma 3.2 Let $X$ be an irreducible, reduced hypersurface in $\mathbb{P}^{n+1}$ of degree greater than 1. Let $P \in X^{\mathrm{sm}}$. Then the family of lines passing by $P$ and contained in $X$ has dimension smaller than $n-1$. 
Proof Assume by contradiction that the dimension is $n-1$. Then $X$ contains a $\mathbb{P}^{n}$ or a cone with vertex $P$. This is not possible because $X$ is irreducible of degree greater than 1 and $P \in X^{\mathrm{sm}}$.

Lemma 3.3 Let $X$ be an irreducible, reduced hypersurface of $\mathbb{P}^{n+1}, n \geq 1$. Assume $Q \in \mathcal{W}(X)$ general. Then the family $\mathcal{V}_{Q}$ has dimension $n-1$.

Proof We will proceed by induction on $n$, starting from the case $n=1$. By applying Lemma 2.16 we know that there must be at least two multi-tangent lines to $X$ and passing by $Q$. If $Q \in \mathcal{W}(X)^{\text {in }}$, notice that one of the two lines must be different from $T_{Q}(X)$, hence the dimension is still 0 . For the general case $n \geq 2$, assume the result is true for $X$ of dimension $n-1$, and prove it for $X$ of dimension $n$. Let $Q \in \mathcal{W}(X)$ be general and assume by contradiction that $\operatorname{dim} \mathcal{V}_{Q}(X)<n-1$. Taking a general hyperplane $H$ through $Q$ we will get $\operatorname{dim} \mathcal{V}_{Q}(X \cap H)<n-2$, and this is in contradiction with the induction hypothesis.

Now we need to describe when the family $\mathcal{V}(X)_{\mathscr{C}}$ is filling, where $\mathscr{C}$ is a curve contained in $\mathcal{W}(X)$.

Proposition 3.4 Let $X$ be an irreducible, reduced hypersurface of $\mathbb{P}^{n+1}, n \geq 2$. Assume $\mathscr{C} \subset \mathcal{W}(X)$ is an irreducible curve which is not contained in a linear space of codimension 2. Then $\mathcal{V}(X)_{\mathscr{C}}$ is filling.

Proof Thanks to Lemma 3.3, we know that $\mathcal{V}_{\mathscr{C}}$ has dimension $n$. It remains to prove that $\mathcal{V}_{\mathscr{C}}$ is dominant. Assume by contradiction that this is not the case, so that $\mathcal{V}_{\mathscr{C}}$ is contained in a finite union of hypersurfaces $V_{j} \subset \mathbb{P}^{n+1}, j=1, \ldots, r$. If we consider $Q \in \mathscr{C}$ general, $\mathcal{V}_{Q}(X)$ is the union of the cones over $X \cap V_{j}$ with vertex $Q$, each of which correspond to $V_{j}$. As a consequence we vary the vertex $Q \in \mathscr{C}, \mathcal{V}_{Q}(X)$ stays the same, so each component $V_{j}$ is constant. Hence, the whole $\mathscr{C}$ is contained in the vertex of each $V_{j}$, and by [1, Proposition 1.3 (i)], also the linear span $\langle\mathscr{C}\rangle$ must be contained in the vertex of each $V_{j}$. Since we are assuming that the codimension of $\langle\mathscr{C}\rangle$ is strictly greater than 2 , the only possibility is that each $V_{j}$ is a linear space. Moreover, since $\mathscr{C}$ is contained in each $V_{j}$, there must be only one of them, so $r=1$, and $\mathscr{C}$ is a curve in a $\mathbb{P}^{n}$.

Assume first that $n>2$, take a general point $Q \in \mathscr{C}$ and a general plane $K \cong \mathbb{P}^{2}$ such that $Q \in K$. By the genericity of the choice of $K$, we know that the family $\mathcal{V}_{Q}(X \cap K)$ is composed by just the line $V_{1} \cap K$. If $Q \in \mathcal{W}(X)^{\text {out }}$, then we just notice that by Lemma 2.16 there must be at least two lines on $\mathcal{V}_{Q}(X \cap K)$, and this gives a contradiction. If $Q \in \mathcal{W}(X)^{\text {in }}$, we have to be more careful due to the way we defined $\mathcal{V}_{Q}(X)$. Since $K$ is general, we know that the tangent line $C_{Q}(X \cap K)$ is simply tangent at $Q$, hence it cannot be one of the two lines described in 2.16, as discussed in Remark 2.15. As a consequence, we get again that there are at least two lines on $\mathcal{V}_{Q}(X \cap K)$, and this gives a contradiction.

We are left with the case $n=2$. In this case, we know that the tangent line $C_{Q}(X \cap K)$ is simply tangent at $Q$ because we chose $Q$ general in $\mathcal{C}$.

Remark 3.5 Consider a curve $\mathscr{C} \subset \mathcal{W}(X)$ and a general line $\ell \in \mathcal{V}(X)_{\mathscr{C}}$. Since the lines of this family are multi tangent at $X$, we have one of the following behaviours 
(C1) The line $\ell$ if is bitangent or asymptotic tangent to $X^{\mathrm{sm}}$;

(C2) The line $\ell$ passes through a point of $X^{\text {sing }}$ and is also tangent at a (necessarily different) point of $X^{\mathrm{sm}}$;

(C3) The line $\ell$ intersects $X^{\text {sing }}$ in more than one point;

(C4) The line $\ell$ is in the tangent cone to $X$ at a point in $X^{\text {sing }}$.

Moreover, from the construction of $\mathcal{V}(X)$ it is clear that all the tangency points are not in $\mathscr{C}$.

We now prove the generalisation of [4, Lemma 4.5]. While the proof follows the same lines, we report it here for the reader convenience to explicitly show that it works for the case of inner non-uniform points.

Lemma 3.6 Let $X$ be an irreducible, reduced hypersurface of $\mathbb{P}^{n+1}, n \geq 2$. Consider the family $\mathcal{V}_{\mathscr{C}}$ associated with a curve $\mathscr{C} \subset \mathcal{W}(X)$. If $\mathscr{V}_{\mathscr{C}}$ has a maximal component of lines of type $(C 1),(C 2)$ or $(C 3)$, then $\mathscr{C}$ is contained in a linear space of codimension 2 in $\mathbb{P}^{n+1}$.

Proof Assume by contradiction $\mathscr{C}$ is not contained in a linear space of codimension 2 in $\mathbb{P}^{n+1}$. Then $\mathcal{V}_{\mathscr{C}}$ is filling by Proposition 3.4. Consider a general $\ell$ in $\mathcal{V}_{\mathscr{C}}$. By construction, there is a point $Q \in \ell \cap \mathscr{C}$. By Lemma 3.3 the family $\mathcal{V}_{Q}$ has dimension $n-1$, so by Lemma $2.4 Q$ is a focal point on $\ell$ of multiplicity at least $n-1$. We know that $\ell$ is multi-tangent at $X$ in points outside $Q$. This comes from the definition of $\mathcal{V}(X)$, so depending on the case in analysis, we can have the following:

(C1) Apply Lemma 2.7. If the line $\ell$ is bitangent to $X^{\mathrm{sm}}$ at two different points, they are foci of multiplicity at least 1 . If the line $\ell$ is asymptotic tangent to $X^{\mathrm{sm}}$, it is a focus of multiplicity at least 2 ;

(C2) By Lemma 2.7, the tangent point of $X^{\mathrm{sm}}$ is a focus of multiplicity at least 1. By a dimension count, there is a subfamily of $\mathcal{V}_{\mathscr{C}}$ of dimension at least 1 of lines passing through the point of $X^{\text {sing }}$. By Lemma 2.4, it is a focus of multiplicity at least 1.

(C3) By a dimension count and by Lemma 2.4, both singular points are foci of multiplicity at least 1 .

In each case, the focal locus on $\ell$ contains the point $Q$ with multiplicity at least $n-1$, and other points which contribute with multiplicity at least 2 . So the degree of the focal scheme on $\ell$ is at least $n+1$, but this contradicts Lemma 2.3.

We are now ready to prove the main result of this paper.

Proof of Theorem 1.3 The case $n=2$ is an easy consequence of the results of [18]. Let now $n>2$ and assume that a component of $\mathcal{W}(X)$ is not contained in a linear space of codimension 2 in $\mathbb{P}^{n+1}$. Consider a curve $\mathscr{C} \subset \mathcal{W}(X)$ with the same property. Thanks to Proposition 3.4 the family $\mathcal{V}_{\mathscr{C}}$ is filling. Let $Q$ be a general point in $\mathscr{C}$ and $\ell$ be the general line in $\mathcal{V}_{Q}$. Since the dimension of $\mathcal{V}_{Q}$ is $n-1$, the point $Q$ is a focus of multiplicity $n-1$ on $\ell$ by Lemma 2.4. As stated in the Remark 3.5, we know that $\ell$ is multi tangent to $X$ in points different from $Q$, which also are part of the focal scheme induced by $\mathcal{V}_{\mathscr{C}}$ on $\ell$. If we are in the cases $(\mathrm{C} 1),(\mathrm{C} 2),(\mathrm{C} 3)$ of Remark 3.5, we can apply Lemma 3.6 and get a contradiction. 
It remains to handle the case $(\mathrm{C} 4)$, namely $\ell$ belongs to the tangent cone of a point in $X^{\text {sing }}$. The family $\widetilde{\mathcal{V}_{\mathscr{C}}}$ is composed by lines joining $\mathscr{C}$ and $X^{\text {sing }}$. By Lemma 2.5 we get that $X^{\text {sing }}$ is the union of a finite number of linear components each isomorphic to $\mathbb{P}^{n-1}$. Moreover, we are assuming the general line belongs to the tangent cone of a point in $X^{\text {sing }}$, hence, in order to have a non-uniform monodromy, the singular locus must split in at least two linear spaces (Proposition 2.11). Let $X_{1}$ be one of the components of $X^{\text {sing }}$, and take a general hyperplane $H \cong \mathbb{P}^{n}$ passing through $X_{1}$. Due to the fact that $\widetilde{\mathcal{V}_{\mathscr{C}}}$ is filling, we can choose $X_{1}$ such that $\mathscr{C}$ is not contained in a hyperplane passing through $X_{1}$, hence, on $H$ there is at least one point $Q \in \mathscr{C}$ not contained in $X_{1}$. If we take $x \in X_{1}$ general, the line $\langle x, Q\rangle$ belongs to the tangent cone $C_{x}(X)$. By Lemma 2.9, there must be a component of the intersection $X \cap H$ passing through $x$ which is different from $X_{1}$, and this is a contradiction.

We can now prove some results that are consequences of the main Theorem. Theorem 1.4 shows that Conjecture 1.2 holds with the additional hypothesis of $X$ being smooth or a general projection of a smooth variety. We report the Bertini theorem as in [16, Theorem 3.3.1] formulated in the context we will need for the proof of Theorem 1.4 .

Theorem 3.7 Let $\tilde{X} \subset \mathbb{P}^{2 n+1}$ a variety of dimension $n$. Let $\Lambda$ be a linear space of dimension $n+k$, for every $k=1, \ldots, n-1$, such that $\tilde{X} \cap \Lambda$ is smooth at every point. Then the section $\tilde{X} \cap H$, for a general $H$ containing $\Lambda$, is irreducible.

Proof The proof of this result is in [16, Theorem 3.3.1]. For the reader convenience we remark that there, the dimension of the ambient space is denoted by $r, \Lambda$ is a linear space of dimension $r-d-1$, with $d<n$.

The proof of Theorem 1.4 relies on a famous result of Mather [17], which is very useful to study the singularities arising from projection maps. The following is an algebraic version which is written here restricting it to the case we will need. The complete version is [2, Theorem 1, Theorem 2].

Theorem 3.8 [2, Theorem 1] Let $\tilde{X} \subset \mathbb{P}^{2 n+1}$ be a smooth variety of dimension $n$. Let $L$ be a linear subspace of dimension $n-1$ such that $L \cap X=\emptyset$. Let $X$ be the image of $\tilde{X}$ under the linear projection $\pi_{L}: \mathbb{P}^{2 n+1} \rightarrow \mathbb{P}^{n+1}$. For any $i \leq n$, define $\tilde{X}_{i}:=\left\{x \in \tilde{X} \mid \operatorname{dim}\left(T_{x} \tilde{X} \cap L\right)=i-1\right\}$. For L general, every $\tilde{X}_{i}$ is smooth and, when not empty, its codimension in $\tilde{X}$ is $i \cdot(i+1)$.

We are now ready to prove Theorem 1.4.

Proof of Theorem 1.4 Fix $n=\operatorname{dim} X$, and assume that $X \subset \mathbb{P}^{n+1}$ is a general projection of a smooth variety $\tilde{X}$. By [11,Section 5], we can assume $\tilde{X} \subset \mathbb{P}^{2 n+1}$. Assume by contradiction that $\mathcal{W}(X)$ is not finite, and denote by $K \cong \mathbb{P}^{k}$ the smallest linear subspace of $\mathbb{P}^{n+1}$ containing an irreducible component of $\mathcal{W}(X)$. We remark that by Theorem 1.3, we have $1 \leq k \leq n-1$. Consider a general $H \cong \mathbb{P}^{k+1}$ containing $K$.

Assume first $X \cap H$ is irreducible. By hypothesis $X$ is reduced, and the base locus of the linear system $X \cap H$ is $X \cap K$. Notice that $\operatorname{dim}(X \cap K)=\operatorname{dim}(X \cap H)-1$, unless $K \subset X$, and so $X \cap H$ is reducible. As a consequence, we get that $X \cap H$ 
is reduced, see for instance [12, Proposition 4.6.1]. Now apply Proposition 3.4 to a curve $\mathscr{C} \subset \mathcal{W}(X)^{\text {out }}$ which spans $K$. Since $\mathscr{C}$ spans a subspace of codimension 1 in $H$, we get that the family of lines in $\mathcal{V}_{\mathscr{C}}$ which belongs to $H$ is filling. Since $X \cap H$ is reduced and irreducible, hypothesis of Theorem 1.3 are satisfied and we immediately get a contradiction.

Now assume $X \cap H$ is reducible. Denote by $L$ the linear space in $\mathbb{P}^{2 n+1}$ which gives the projection $\pi_{L}: \tilde{X} \rightarrow X$. Let also $\tilde{H}:=\langle L, H\rangle$, and $\tilde{K}:=\langle L, K\rangle$. Notice that $\tilde{K} \cong \mathbb{P}^{k+n}$ is a suitable $\Lambda$ for applying 3.7. Since we are assuming $X \cap H$ is reducible, we get that $\tilde{X} \cap \tilde{K}$ must be not reduced. This means that $T_{x} \tilde{X} \subset \tilde{K}$ for $x \in \tilde{X} \cap \tilde{K}$. As a consequence, the dimension of $T_{x} \tilde{X} \cap L$ in $\tilde{K}$ is $n-k-1$. From the fact that $1 \leq k \leq n-1$, we have $n-k-1 \geq 0$. This shows that the points of $\tilde{X} \cap \tilde{K}$ are contained in $\tilde{X}_{n-k}$, defined in Theorem 3.8. Now, $\tilde{X} \cap \tilde{K}$ has dimension at least $k-1$ and $\tilde{X}_{n-k}$ has dimension $n-(n-k)(n-k+1)$. We get

$$
\begin{aligned}
k-1 & \leq n-(n-k)(n-k+1) \\
0 & \leq 1-(n-k)^{2}
\end{aligned}
$$

It follows that $k=n-1$, and so $\operatorname{dim}(\tilde{X} \cap \tilde{K})=\operatorname{dim} \tilde{X}_{1}$. Since $\tilde{X}_{1}$ is smooth by Theorem 3.8, this gives a contradiction.

Theorem 1.5 is a consequence of the following result about transpositions in the monodromy group associated with non-uniform points. The idea is to try to study the situation arising from possible counterexamples of Conjecture 1.2.

Proposition 3.9 Let $X$ be an irreducible, reduced hypersurface of $\mathbb{P}^{n+1}$. Assume $\mathcal{W}(X)$ is not finite, and $X$ is not a cone, then the monodromy group associated with all but finitely many points of $\mathcal{W}(X)$ contains transpositions.

Proof This result has been proved for outer non-uniform points in [4, Theorem 1.4]. For simplicity here we will restrict to the case of inner non-uniform points. Let $\mathscr{C}$ be a curve inside $\mathcal{W}(X)^{\text {in }}$ and let $\mathcal{X}_{Q}$ be the family of lines through a point $Q \in \mathscr{C}$ that are tangent to $X$ at smooth points and not lying in the tangent hyperplane to $X$ at $Q$. Let $\mathcal{X}$ be the union of $\mathcal{X}_{Q}$ for every $Q \in \mathscr{C}$.

Let $X^{*} \subset\left(\mathbb{P}^{n+1}\right)^{*}$ be the dual variety of $X$ and let $r$ be the dimension of $X^{*}$. If $X$ is not a cone, by [20, Theorem 1.25] we have that $X^{*}$ is not contained in a hyperplane. Consider the family of hyperplanes $\left\{H_{Q}^{\vee}\right\}$ in $\left(\mathbb{P}^{n+1}\right)^{*}$ dual to the points of $Q \in \mathscr{C}$.

If $H_{Q}^{\vee}$ is general in this family, $\operatorname{dim}\left(X^{*} \cap H_{Q}^{\vee}\right)=r-1$. Since every point of $X^{*}$ is contained in one of such hyperplanes, by dualization we get that the tangent hyperplane to $X$ at a general point pass through the general point of $\mathscr{C}$. As a consequence, the general $Q \in \mathscr{C}$ is contained in a $r-1$ dimensional family of tangent hyperplanes to $X$. The general element of this family is tangent to $X$ along a subvariety of dimension $n-r$ and every line joining $Q$ and this subvariety belongs to $\mathcal{X}$. In particular, the dimension of the family $\mathcal{X}$ is $n$, and the general line in $\mathcal{X}$ is tangent at the general point of $X$.

We still need to show that $\mathcal{X} \rightarrow \mathbb{P}^{n+1}$ is dominant. If this were not the case, the general line of $\mathcal{X}$ would be contained in a finite union of hypersurfaces $V_{i}$, hence it 
would not be tangent at the general point of $X$. Notice that $V_{i}$ cannot coincide with $X$ itself since $X$ is not a cone.

Consider now the focal scheme of $\mathcal{X}$ on $\ell$. Since $Q$ in $\ell$ is a point of multiplicity $n-1$, it gives by Lemma 2.4 a focus of multiplicity $n-1$. Moreover, assume that the general line $\ell$ of $\mathcal{X}$ is not simply tangent to $X$. Then, we would have on $\ell$ at least two focal points of degree one, or at least one focal point of degree 2 . This contradicts Lemma 2.3.

As a consequence, if $X$ is not a cone, we can find simple tangent lines to $X$ passing through the general point of $\mathcal{W}(X)^{\text {in }}$, that correspond to transpositions in the monodromy group.

We can prove Theorem 1.5:

Proof of Theorem 1.5 Proposition 3.9 implies that, if $\mathcal{W}(X)$ is not finite and $X$ is not a cone, then for all but finitely many points there exist a transposition in the monodromy group. As a consequence, for all but finitely many points $Q \in \mathcal{W}(X)$, the field extension given by the projection from $Q$ is not Galois, since the action of an element in the Galois group on a general fibre has no fixed components. Thus, the locus of Galois points is finite.

Moreover, if the map $\pi_{P}$ is indecomposable and the monodromy group of $\pi_{P}$ contains a transposition, then the point $P$ is uniform, see Remark 2.12. Thus, if $P \notin X$ and $\operatorname{deg}(X)$ is prime, then the map $\pi_{P}$ is indecomposable and so $\mathcal{W}(X)^{\text {out }}$ is finite. If $P \in X^{\mathrm{sm}}$ and $\operatorname{deg}(X)-1$ is prime, then the map $\pi_{P}$ is indecomposable and so $\mathcal{W}(X)^{\text {in }}$ is finite.

We conclude this section by some considerations on Conjecture 1.2, which at the moment is the state of the art generalisation of [4, Remark 4.11].

Corollary 3.10 The only class of hypersurface which could provide a counterexample to conjecture 1.2 is given by irreducible and reduced hypersurfaces $X$ in $\mathbb{P}^{n+1}$, which are not cones, and such that there exists a $\mathbb{P}^{k}, 0<k<n$, where $X \cap \mathbb{P}^{k+1}$ is reducible for every $\mathbb{P}^{k+1} \supset \mathbb{P}^{k}$.

Proof By Theorem 1.3 the non-uniform locus $\mathcal{W}(X)$ is not finite and contained in a linear subspace $\langle\mathcal{W}(X)\rangle \cong \mathbb{P}^{k}$ with $k<n$. Consider a general $H \cong \mathbb{P}^{k+1}$ such that $H \supset \mathbb{P}^{k}$. The section $X \cap H$ is reduced since $X$ is. If moreover $X \cap H$ is irreducible, then we get a contradiction to Theorem 1.3 , since $\mathcal{W}(X)=\mathcal{W}(X \cap H)$ is in codimension one in $H$.

Acknowledgements The authors are members of GNSAGA of INdAM. During the preparation of the paper, the authors were partially supported by PRIN 2017 Moduli and Lie theory, and by MIUR: Dipartimenti di Eccellenza Program (2018-2022) - Dept. of Math. Univ. of Pavia. We would like to thank Gian Pietro Pirola for the helpful discussions and suggestions during the preparation of the paper. We are also grateful to Alberto Albano and Francesco Russo for the help in understanding how to remove the condition (C4) from the proof of the main result.

Open Access This article is licensed under a Creative Commons Attribution 4.0 International License, which permits use, sharing, adaptation, distribution and reproduction in any medium or format, as long as you give appropriate credit to the original author(s) and the source, provide a link to the Creative Commons licence, 
and indicate if changes were made. The images or other third party material in this article are included in the article's Creative Commons licence, unless indicated otherwise in a credit line to the material. If material is not included in the article's Creative Commons licence and your intended use is not permitted by statutory regulation or exceeds the permitted use, you will need to obtain permission directly from the copyright holder. To view a copy of this licence, visit http://creativecommons.org/licenses/by/4.0/.

\section{References}

1. Ådlandsvik, B.: Joins and higher secant varieties. Math. Scand. 61, 213-222 (1987)

2. Alzati, A., Ballico, E., Ottaviani, G.: The theorem of Mather on generic projections for singular varieties. Geom. Dedicata 85, 113-117 (2001)

3. Cifani M.G.: Monodromy of general hypersurfaces, preprint arXiv:2007.09958, (2020)

4. Cifani, M.G., Cuzzucoli, A., Moschetti, R.: Monodromy of projections of hypersurfaces. Ann. Mat. Pura ed Appl. 1923, 1-18 (2021)

5. Ciliberto, C., Flamini, F.: On the branch curve of a general projection of a surface to a plane. Trans. Amer. Math. Soc. 363, 3457-3471 (2011)

6. Cukierman, F.: Monodromy of projections. Mat. Contemp. 16, 9-30 (1999)

7. Cuzzucoli, A., Moschetti, R., Serizawa, M.: Non-uniform projections of surfaces in $\mathbb{P}^{3}$. Le matematiche, LXXII (2017)

8. Fukasawa, S.: Complete determination of the number of Galois points for a smooth plane curve. Rend. Semin. Mat. Univ. Padova 129, 93-113 (2013)

9. Fukasawa, S.: Automorphism groups of smooth plane curves with many Galois points. Nihonkai Math. J. 25, 69-75 (2014)

10. Fukasawa, S., Takahashi, T.: Galois points for a normal hypersurface. Trans. Amer. Math. Soc. 366, 1639-1658 (2014)

11. Fulton, W., Lazarsfeld, R.: Connectivity and its applications in algebraic geometry. Algebraic Geom. (Chicago. Ill) 862(1981), 26-92 (1980)

12. Grothendieck, A.: Éléments de géométrie algébrique. IV. Étude locale des schémas et des morphismes de schémas. II, Inst. Hautes Études Sci. Publ. Math., (1965), p. 231

13. Harris, J.: Galois groups of enumerative problems. Duke Math. J. 46, 685-724 (1979)

14. Harris, J.: Algebraic geometry, Graduate Texts in Mathematics, 133 (1995)

15. Isaacs, I.M.: Finite group theory. Graduate Studies in Mathematics, vol. 92. American Mathematical Society, Providence, RI (2008)

16. Lazarsfeld, R.: Positivity in algebraic geometry. I, Ergebnisse der Mathematik und ihrer Grenzgebiete. 3. Folge., 48 (2004)

17. Mather, J.N.: Generic projections. Ann. Math. 2(98), 226-245 (1973)

18. Pirola, G.P., Schlesinger, E.: Monodromy of projective curves. J. Algebraic Geom. 14, 623-642 (2005)

19. Segre, C.: Un' osservazione sui sistemi di rette degli spazi superiori. Rendiconti del Circolo Matematico di Palermo, Tomo II, 148-349 (1888)

20. Tevelev, E.A.: Projectively dual varieties. J. Math. Sci. (N.Y.) 117, 4585-4732 (2003)

21. Yoshihara, H.: Galois points for smooth hypersurfaces. J. Algebra 264, 520-534 (2003)

Publisher's Note Springer Nature remains neutral with regard to jurisdictional claims in published maps and institutional affiliations. 